\title{
Tactile roughness of grooved surfaces: The touching process and effects of macro- and microsurface structure*
}

\author{
SUSAN J. LEDERMAN $\dagger$ \\ University of Toronto and Defence and Civil Institute of Environmental Medicine \\ Toronto, Ontario, Canada
}

\begin{abstract}
Ss made magnitude estimates of the perceived roughness of grooved aluminum plates. Two aspects of the touching process were altered and their effects upon roughness examined. Roughness increased with increasing finger force, regardless of whether the $S$ or the $E$ chose the values. Rate of hand motion had a negligible effect on perceived roughness, indicating a roughness constancy and providing further evidence of the relative unimportance of vibratory frequency. The effects of these components of the touching process were discussed in terms of an active-passive continuum rather than a dichotomy. Perceived roughness declined with increasing land width (with narrow grooves), although only over the widest half of the land range; there was no land effect when the grooves were wide. In addition to these macrostructural parameters, the effects of two stimulus production techniques were compared. The discrepancies between the two sets of data were interpreted in terms of the microscopic irregularities of the plate surfaces. The findings were briefly related to an analysis of perceived roughness of grooved surfaces in terms of static deformation of the skin.
\end{abstract}

Roughness is one of the most prominent aspects of tactile texture, yet little systematic research in the area exists. Katz (1925), one of the earliest investigators, published an extensive monograph which examined the perception of texture by both active and passive touch [or by obtained and imposed stimulation, respectively, to use Gibson's (1966) distinction]. Although primarily phenomenological, the work does include cursory reference to several experiments on perceived roughness. Highlights of the monograph, which remains untranslated at this time, may be found in Zigler (1926) and in Krueger (1970). For example, Katz found that "... light pressure gives a more pronounced smoothness than strong pressure at all rates of movement [Zigler, p. 331]." In this experiment, he moved paper surfaces across the Os' fingers at several different speeds.

Regarding the nature of the surface being touched, Stevens and Harris (1962) found that perceived roughness (magnitude estimates) of sandpapers increases with decreasing grit number (which is proportional to the inverse of the particle diameter).

\footnotetext{
*This paper is based on part of a thesis submitted to the University of Toronto in partial fulfillment of the requirements for the PhD degree. I wish to thank R. G. Harris of Sir George Williams University for his criticisms of the manuscript, and $\mathbf{M}$. $M$. Taylor of DCIEM for his comments on the research.

†Now at Queen's University. Address reprint requests to Queen's University, Department of Psychology, Kingston, Ontario, Canada K7L 3N6.

t†This is DCIEM Research Paper No. 73-RP-965.
}

However, the authors were more inerested in the psychophysical function than in roughness per se.

More recently, Lederman and Taylor (1972) made an attempt to define systematically some stimulus parameters relating to the percept of "roughness." Ss made magnitude estimates of the perceived roughness of grooved aluminum plates by actively moving their middle three fingers across the surfaces under conditions which controlled finger force. The results indicated that apparent roughness tends to increase as the grooves widen, as the finger force increases, and as the spacing between the grooves ("land") narrows. The reader is cautioned to avoid comparing in detail the growth functions of the Lederman and Taylor (1972) and Stevens and Harris (1962) papers, since Ekman, Hosman, and Lindström (1965) found in their scaling study that the slopes of the psychophysical functions for roughness varied across Ss from .8 to 3.5. In this last study, the physical stimulus parameter was coefficient of friction, and the stimulus materials included various grades of sand paper, cardboard, and writing paper.

The present paper has several aims. The first is to examine the touching process, a fundamental aspect of the perception of roughness, or more generally, of texture. Taylor, Lederman, and Gibson (1973) conceive of the touching process in the perception of texture as consisting of a number of components or ways in which a surface may be explored. For example, an individual may choose the force, rate and direction of touching motions, as well as the position 
of skin-surface contact. Any, or all, of these aspects may be varied over time since feedback is considered to be an important characteristic of the touching process. The authors postulate three fundamental behavioral feedback loops:

"The major one is an overall control loop (i) whose function is to carry out the policy decision to look for a certain feature of the texture, such as roughness or elasticity. The original intention to touch something, to investigate an aspect of texture such as roughness, is taken to be a command to the major loop control element, labeled Behavior Control. The function of this module is to select a touching strategy adequate for the job. In the case of roughness, the appropriate mode of touching involves a light sweeping motion of the fingertips back and forth over the surface. Probing for subsurface objects in an elastic medum, such as a pea under a foam mattress, requires an entirely different mode of touching.

"The secondary loop (ii) has the function of executing the individual motions required to implement the desired touching mode. Its commands are produced by the element labeled Movement Control. This element breaks down the general command from the Behavior Control into a sequence of specific motion commands, which go to the control element of the innermost loop (iii) labeled Motor Control. This is the familiar kinaesthetic control loop, which breaks down the individual movement commands into muscle commands and monitors the effects to ensure that the movement command is properly executed [p. 265-267]."

Thus the planning of the touching activity is carried out at two levels by the strategy (i) and tactics (ii) control loops, while the actual movement execution is handled by the third, most deeply nested motor control loop (iii). Such a conception of the touching process leads to a reformulation of Gibson's active-passive distinction because active and passive touch are now considered to lie along a continuum. Total "activeness" is defined as a person's having complete control over all three feedback loops, and thus over both the planning and execution process, whereas at the other extreme, passive touch deprives the individual of all such control. From Gibson's point of view, the Lederman and Taylor (1972) study involves active touch, and the Katz study (1925), passive touch. However, according to the Taylor et al approach, one must say that in neither study were Ss totally active. Lederman and Taylor's Ss executed the touching motions and forces, but had no control over the particular values of the force component. Katz's Ss, in addition, had no control over the execution of the touching motions. Taylor et al (1973) suggest that experiments which interfere with the individual's control of the touching process by blocking various aspects of the three feedback loops may help to clarify the nature of this active-passive continuum in the perception of texture. An earlier study (Lederman \&
Taylor, 1972) showed that perceived roughness was affected by the fingertip force exerted by the $S$ when the force value was chosen by the E. Experiment I extends this study by asking whether apparent roughness would differ in terms of discrimination and/or response precision if Ss were permitted to choose finger force. Experiment II examines the role of a second intuitively important aspect of the touching process in the perception of roughness, the rate of hand motion. This study is of additional interest in that it permits a further evaluation (see Lederman \& Taylor, 1972) of the contribution of mechanical vibratory frequency to the perception of roughness.

A second aim of the paper is to extend the results of the Lederman and Taylor (1972) study concerning the significance of surface structure to the apparent roughness of grooved aluminum plates. In addition to assessing the relative contributions of certain groove dimensions, the effects of two different plate production techniques are also evaluated. The results of Experiments I, III, and IV indicate, in general, that any analysis of surface structure and its contributions to perceived roughness must take place at both macro- and microscopic levels of analysis. Such considerations highlight some of the difficulties encountered in producing stimulus surfaces for use in tactile perception experiments. It is, in fact, possible that inadequate production techniques is one of the major reasons that tactile perception remains relatively unexplored. The production methods outlined in the present paper, then, contribute some information to our current technical knowledge of tactile stimulus production.

A third and final aim of the paper is to provide experimental data for use in a mechanical analysis (Taylor \& Lederman, 1974) of the skin resting on grooved aluminum surfaces. This analysis represents an attempt to determine some one (or more) parameter(s) of skin deformation which can predict changes in perceived roughness resulting from alteration of groove width and finger force, the most influential factors in the present experiments. The effect of land width, which is ambiguous and far less significant, is only considered qualitatively in the analysis.

\section{THE TOUCHING PROCESS}

\section{Experiment I}

The Lederman and Taylor (1972) study indicated that when Ss are required to alter finger force, their perception of the roughness of grooved surfaces changes. However, it is not known whether people actually adjust force when tactually exploring a surface, and, more to the point, whether their perceptions change concomitantly with such adjustments. Experiment I deals with the following questions. Will a person vary finger force if free to do 
so, and under what conditions? Will his perception of surface roughness change with the force he applies? Will "controlled" and "free" force conditions yield similar or at least compatible results with respect to the growth function for roughness and/or response precision? The last question concerns the effect on perceived roughness of interfering with the strategy and tactics control loops mentioned earlier.

Ss made magnitude estimates of roughness in two controlled force conditions, $1 \mathrm{oz}(\approx 28 \mathrm{~g})$ (called "low") and $16 \mathrm{oz}(\approx 448 \mathrm{~g})$ (called "high"), and in a third where they were permitted to press however hard they wished ("normal").

\section{Method}

Subjects. The details concerning Ss used in each of the four experiments are reported in Table 1 .

Apparatus and Stimuli. The apparatus was the same as that described by Lederman and Taylor (1972). Its design was similar to a classical balance scale, its purpose being to control finger force. It was possible to add weights to one end of the balance arm so that the $S$ was required to exert an equal counterbalancing force as he moved his hand across the stimulus surface in a tray at the other end. The constancy of the force was altered only by small acceleration forces caused by the slight up-and-down motions of the balance arm which occurred during the movement of the finger across the stimulus surfaces. However, as mentioned earlier (Lederman \& Taylor, 1972), these variations were no more than about $20 \%$ of the nominal value.

The force apparatus was modified for the present experiment in the following manner. When a freely applied force was to be measured, a force gauge (Hunter Spring Co., Model LO-2M) was placed under the balance arm, $5 \frac{1}{2} \mathrm{in}$. from the fulcrum of the balance apparatus. In this position, the force gauge registered the fingertip force multiplied by the appropriate lever ratio.

To filter out high-frequency variations in the reading of the force gauge and to provide relatively long-term averaging, a dashpot was attached between the balance arm and the movable base at the front of the apparatus. A dashpot is a viscous damping mechanism which will not move rapidly even under large forces, but which moves easily at low speed even under very small forces. It greatly
Table 1

Subject Information for Experiments I, II, III, and IV

\section{Experiment I}

Twelve right-handed (as defined by preferred writing hand) Ss, five males and seven females participated. They were all employees serving in various capacities at DCIEM. Three of the Ss had been in a similar psychophysical experiment; all others were experimentally naive. Their ages ranged from 17 to 51 years.

\section{Experiment II}

Six paid right-handed Ss were used. There were two males and four females, their ages ranging from 16 to 24 years. All had previously participated in a number of experiments of this kind.

\section{Experiment III}

Four paid Ss, two males and two females, participated. Their ages ranged from 16 to 26 years. All had previous experience in such experiments.

\section{Experiment IV}

All Ss from Experiment II were paid for participating in Experiment IV.

reduced large and rapid fluctuations in the position of the force gauge indicator which were due to small motions of the balance arm rather than to deliberate changes in S's finger force. When the dashpot and force gauge were disconnected, the apparatus controlled finger force exactly as described by Lederman and Taylor (1972).

The stimuli were smoothed aluminum alloy plates, $51 / 2 \times 41 / 2 \times$ $3 / 16$ in., each with a set of linear grooves cut parallel to the line of the balance arm, and extending across the width of the central third of the plate (see Lederman \& Taylor, 1972, Fig. 1). The plates fit at one end of the balance arm into a stimulus tray, the position of which could be adjusted along the arm to accommodate variations in $\mathrm{Ss}^{*}$ arm lengths. Two duplicate sets of tiles, each containing eight plates of different groove widths but fixed land width, were used. One set (GA) was used in the Lederman and Taylor (1972) study, while the second $(\mathrm{GB})$ was newly produced. The dimensions ${ }^{1}$ and names of the plates used in all four experiments are presented in Table 2. The names use an alphanumeric code in which the letters

Table 2

Dimensions and Names of the Stimulus Plates Used in Experiments I, I, III, and IV

Groove Width

\begin{tabular}{|c|c|c|c|c|c|c|c|c|c|}
\hline $\begin{array}{l}\text { Land } \\
\text { Width }\end{array}$ & $\begin{array}{c}5(\sim .125 \\
\mathrm{mm})\end{array}$ & $\begin{array}{c}7(\sim .175 \\
\mathrm{mm})\end{array}$ & $\begin{array}{c}10(\sim .25 \\
\mathrm{mm})\end{array}$ & $\begin{array}{c}15(\sim .375 \\
\mathrm{mm})\end{array}$ & $\begin{array}{c}20(\sim .50 \\
\mathrm{mm})\end{array}$ & $\begin{array}{c}25(\sim .625 \\
\mathrm{mm})\end{array}$ & $\begin{array}{c}30(\sim .75 \\
\mathrm{mm})\end{array}$ & $\begin{array}{c}35(\sim .875 \\
\mathrm{mm})\end{array}$ & $\begin{array}{c}40(\sim 1.0 \\
\mathrm{mm})\end{array}$ \\
\hline $\begin{array}{l}15(\sim .375 \mathrm{~mm}) \\
20(\sim .50 \mathrm{~mm}) \\
25(\sim .625 \mathrm{~mm}) \\
30(\sim .75 \mathrm{~mm}) \\
35(\sim .875 \mathrm{~mm}) \\
40(\sim 1.0 \mathrm{~mm})\end{array}$ & $\begin{array}{l}\mathrm{Ia}^{*} \text { (GA5) } \\
\mathrm{Ib} \text { (GB5) } \\
\mathrm{IV}^{*}(\mathrm{G} 5)\end{array}$ & II (G7) & $\begin{array}{l}\text { III (L7) } \\
\text { IA* (GA10) } \\
\text { Ib (GB10) } \\
\text { II (G10) } \\
\text { III (L10) } \\
\text { IV (G10) } \\
\text { III (L15) } \\
\text { III (L20) } \\
\text { III (L25) } \\
\text { III (L30) } \\
\text { III (L35) } \\
\text { III (L40) }\end{array}$ & $\begin{array}{l}\text { Ia* (GA15) } \\
\text { Ib (GB15) } \\
\text { II (G15) }\end{array}$ & $\begin{array}{l}\mathrm{Ia}^{*}(\mathrm{GA} 20) \\
\mathrm{Ib}(\mathrm{GB} 20) \\
\mathrm{II}(\mathrm{G} 20) \\
\mathrm{IV}(\mathrm{G} 20)\end{array}$ & $\begin{array}{l}\text { III (WL7) } \\
\text { Ia* (GA25) } \\
\text { Ib (GB25) } \\
\text { II (G25) } \\
\text { III (WL10) } \\
\text { IV (G25) } \\
\text { III (WL15) } \\
\text { III (WL20) } \\
\text { III (WL25) } \\
\text { III (WL30) } \\
\text { III (WL35) } \\
\text { III (WL40) }\end{array}$ & $\begin{array}{l}\text { Ia (GA30) } \\
\text { Ib (GB30) } \\
\text { II (G30) } \\
\text { IV (G30) }\end{array}$ & $\begin{array}{l}\text { Ia (GA35) } \\
\text { Ib (GB35) } \\
\text { II (G35) } \\
\text { IV (G35) }\end{array}$ & $\begin{array}{l}\text { Ia (GA40) } \\
\text { Ib (GB40) } \\
\text { II (G40) } \\
\text { IV (G40) }\end{array}$ \\
\hline
\end{tabular}

Note-All plates in Experiment $I$ are $S$ plates. All plates in Experiments II, III, and IV are EDM plates with the exception of one plate in Experiment IV (groove $=.005 \mathrm{in}$., land $=.010 \mathrm{in.}$ ). Ia and Ib refer to the old and duplicate sets, respectively, used in Experiment I. Groove width and land width are in thousandths of an inch; metric units are in parentheses.

${ }^{*}$ Groove depth $=.005 \mathrm{in} .(\sim .125 \mathrm{~mm})$; all other $S$ plates have a groove depth of $.010 \mathrm{in} .(\sim .25 \mathrm{~mm})$. The groove depth of the EDM plates corresponds to the groove width. 
represent the dimension varied, and the number its value in thousandths of an inch.

Experimental Procedure. The procedure to be described is the same as that detailed in the Lederman and Taylor (1972) paper, and applies to the remaining experiments reported here. The major procedural change in the four present experiments was that the middle finger was used rather than three fingers, to permit more precise control of finger force. With the use of three fingers, there is no guarantee that the force applied was equally distributed across all fingers. $S$ sat beside the apparatus and placed his right elbow in a shaped, swivelled armrest mounted directly over the balance arm fulcrum. He was instructed to move the tip of his finger across the surfaces (at right angles to the balance arm) at any single rate he wished, provided he maintained that rate at all times. On the controlled force trials, he was told to press down on the surface with sufficient force to keep the balance arm continually steady and level. Intermixed with controlled force trials would be others during which he would be allowed to feel the surface "normally," i.e., he could press however hard he wished. However, S was asked to maintain a constant force once he had chosen one. On "normal" trials, the dashpot and force gauge were set in place, thereby severely restricting the amount of up-down motion of the balance arm. However, as it was necessary to wait until the gauge indicator had stabilized, the intertrial interval was considerably longer for those trials. The average force applied to a plate was recorded on each trial. If $\mathrm{S}$ altered his force to a second stable value, the average of the two forces was recorded. Such occasions indicated a change of stable force chosen by $\mathrm{S}$.

A modified magnitude estimation procedure, identical to that reported by Lederman and Taylor (1972), was used in all experiments. $S$ was told that he must assign numbers which corresponded to the roughness of the surfaces, but to ignore any changes in finger force (or hand speed, as in Experiment II) when making his judgments. Neither standard nor modulus was used. Rather, before the experiment began, $S$ was presented with two practice plates. $E$ told $S$ that these should give him an idea of the range of roughnesses involved, and thus permit him to choose a range of numbers which could include any positive (nonzero) value. Examples of possible range values, such as 1 to 10 and 10 to 100 , were suggested to each S. ${ }^{2}$ It was emphasized that there was an infinite set of numbers between 0 and 1 . The two practice plates were always what felt the roughest and smoothest to $E$, although they were described to the Ss as "... one of the roughest and one of the smoothest...." Ss were encouraged to extend their numerical responses beyond the range chosen if they felt plates which were smoother or rougher than the practice plates.

Experimental Design. Twelve Ss judged the roughness of eight plates of various groove widths, for each of three different force conditions, with two replications of these 24 trials per day, and two separate sessions (days). Two duplicate sets were used, but only one per day. Half of the Ss were presented with Set GA on Day 1 and with Set GB on Day 2, while the remaining Ss judged the plate sets in the reverse order. The presentation order for stimulus plates (within a set) and forces was selected according to a controlled randomization procedure.

\section{Results}

Analyses of variance were performed on the magnitude estimate scores after logarithmic transformation. In the first analysis, a completely crossed, five-factor design was used: Subjects (12) by Tile Set (2) by Replication (2) by Groove Width (8) by Fingertip Force (3). Increasing groove width resulted, in a significant increase in perceived roughness $(\mathrm{p}<.001)$, as did finger force $(\mathrm{p}<.001)$. In Fig. 1 , perceived roughness (log magnitude estimate) is plotted as a function of groove width (log scale) for the three force conditions. Only geometric means are shown. The data from the two tile sets were combined since the effects of tile set and the interaction term,
Tile Set by Force, were not significant. Although the new set of tiles, GB, resulted in higher estimates than the old set, the Tile Sets by Groove Widths interaction term was small and only just approaching significance at the .05 level. The shapes of the combined force functions are generally concave, with a relatively flat portion at the narrow groove end. In the experiments to follow, the reader should assume, unless otherwise mentioned, that effects due to groove width and controlled force are always significant, usually at the .001 level.

An analysis of variance of variance, or ANOVAVA as referred to by Lederman and Taylor (1972; see Appendix), permits one to determine whether the precision of the Ss' responses differed for the controlled and uncontrolled force conditions. The results of such an analysis, however, failed to show a significant difference.

An analysis of variance was repeated this time with days substituted in place of the tile set factor. This was necessary since the two terms were not completely crossed and the analysis of variance program available could not handle crossover designs. Possible main effects for days and replications could not be meaningfully interpreted since Ss were permitted to change their ranges between days and replications. The higher-order interaction terms involving these factors can be used to evaluate the effects of days and replications. However, none was significant in the present experiment, nor were any others involving days or replications in the three other experiments reported here.

Along with the estimates of roughness, the average "normal" fingertip forces (in ounces) to the nearest quarter of an ounce were recorded. These scores were fairly imprecise estimates, since the individual data points from which the averages were calculated

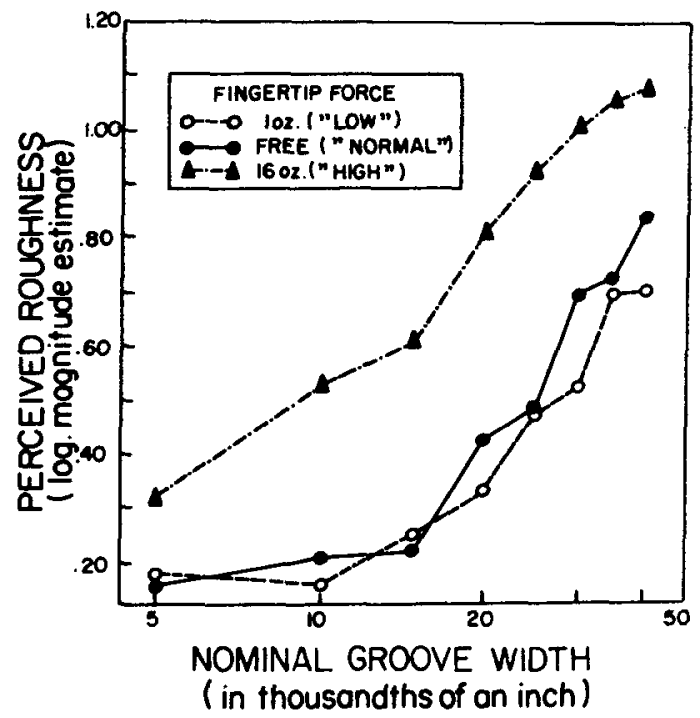

Fig. 1. Experiment I: Perceived roughness as a function of groove width uner controlled and "free" force conditions. 
represented not single force values, but rather ranges of values. Using the logarithmically transformed "free" ("normal") force values as dependent variable instead of roughness, two analyses of variance were performed with four completely crossed factors: subjects, days (or tile sets), replications, and groove widths. In the uncontrolled touching situation, fingertip force tended to increase with increasing groove width $(\mathrm{p}<.01)$ and the Tile Sets by Groove Widths interaction term was also highly significant $(p<.005)$. There were large $S$ differences in the forces applied; the averages for Ss ranged from $\sim .61$ to $\sim 6.3 \mathrm{oz}(\sim 17$ to $\sim 172 \mathrm{~g})$, with an overall average of $-2.44 \mathrm{oz}(\sim 70 \mathrm{~g})$. Figure 2 shows the relation between $\log$ (free fingertip force) and groove width (log scale) for the old (GA) and new (GB) sets. There is a great deal of variability, as would be expected with the relatively imprecise (and inexpensive) technique used for the initial research here; however, there does appear to be a trend in both funtions, finger force tending to increase slightly with increases in groove width. The reason for the anomalously low force on Plate GB25 of the new set is not clear, although it occurs in approximately two-thirds of the sessions, and appears largely responsible for the significant Groove Widths by Tile Set interaction.

\section{Discussion}

Each of the three force functions shown in Fig. 1 is concave upwards, with a relatively flat portion at the narrow groove end of the scale. This contrasts with the Lederman and Taylor (1972) results, where the functions are nearly linear thoughout their range. The reasons for the difference may well relate to the change in the number of fingers used for touching. Research on this issue is being planned; however, at present no complete explanation can be provided. The uncontrolled force condition represents a more "active" touching condition, according to Taylor et al (1973), than the 1 - and $16-\mathrm{oz}(\approx 28$ and $\approx 448 \mathrm{~g}$ ) force conditions, since it does not interfere with the tactics control loop in the choice of force values. However, the free force function, which is described by an average value of approximately $2.44 \mathrm{oz}(\approx 70 \mathrm{~g})$, falls between the 1- and 16-oz curves, as one might expect if it were to represent an identical controlled force value. Under the present experimental conditions, then, both controlled and uncontrolled force would appear to have similar effects on perceived roughness estimates of grooved plates. Furthermore, the results of the ANOVAVA analysis indicate that there was no difference between the more and less active force conditions with respect to response precision. It was originally thought that the more active free force condition might result in more highly precise responses, even if the growth functions were not significantly different. It appears, therefore, that the increased activeness provided the $S$ by allowing him to choose his own force value altered neither the

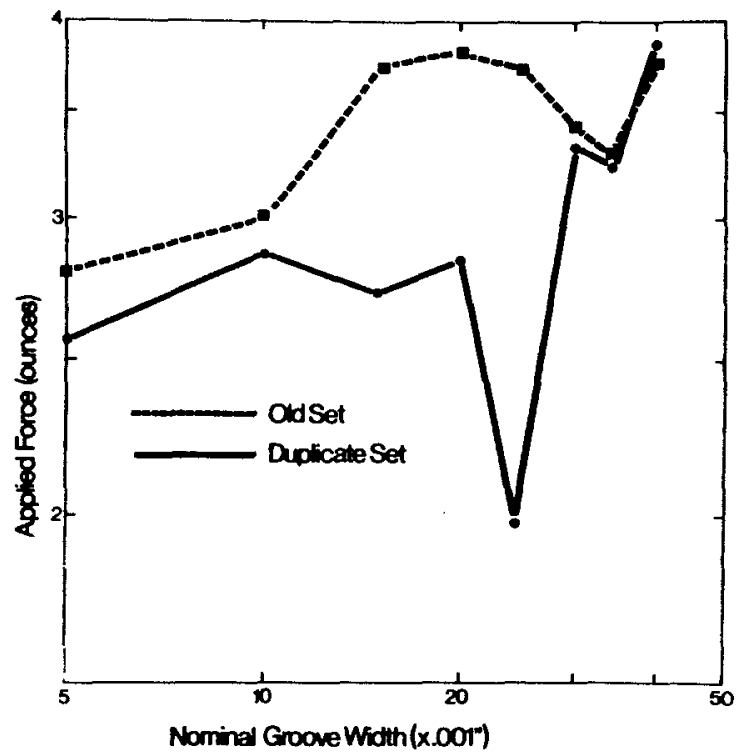

Fig. 2. Experiment I: Average "free" force (log scale) as a function of groove width and tile set.

roughness growth function nor response precision compared to controlled force conditions.

Regarding the question posed earlier concerning the conditions under which Ss may alter their finger force, Ss tended to press harder as the width of the grooves was increased, although this effect was small. One possible reason for this might simply be that greater force must be exerted to prevent the skin from catching on the leading edge of each land. It would be of interest to perform a further experiment to determine whether perceived roughness increases with increasing force when groove width is held constant.

\section{Experiment II}

As part of his studies, Katz (1925) emphasized the importance of movement between the hand and the surface: "...to feel modifications of the surface (hardness, graininess, etc.) and thus to recognize the specific material, movement is necessary [Krueger, 1970, p. 339]." Meenes and Zigler (1923) also found that relative motion between hand and object was necessary for the perception of roughness and smoothness. Can one then ask whether the rate at which a person moves his fingers across a surface affects the perceived roughness of that surface? When we have Ss alter the speed of motion, another component of the touching process, we are again interrupting the tactical loop of the motor control system. To this extent, we have made the touching behavior somewhat less active as conceived by Taylor et al (1973).

In addition to evaluting rate of hand motion in the perception of roughness, there is a second significant concern of the present experiment. To Katz, movement is important because it produces vibratory sensations. What role does vibration play in the 
experience of roughness? As discussed elsewhere (Taylor et al, 1973), "Variations in the perception of roughness may depend on the overall energy of the vibration modulated by the sensitivity characteristic of the sensors (as loudness depends on the energy of the sound and on the spectral sensitivity of the ear), or it may depend on the vibratory frequency ... [p. 257]." Taylor and Lederman (Taylor et al, 1973) emphasize the energy characteristics of the vibratory signal at frequencies to which the finger is sensitive, while Gibson supports the frequency-dependent interpretation.

Lederman and Taylor (1972) provide evidence to support the energy-dependent explanation. Two sets of aluminum plates with parallel linear grooves along the surface were used. In one set, different groove widths were used while land width was held constant, whereas in the second set, land width varied and groove width was constant such that the dimensions were exactly reversed. By maintaining hand speed relatively constant, it was possible to manipulate the frequency of vibratory impulses identically in both sets; the vibratory frequency varies inversely with the sum of the widths of a groove and a land, if one defines the vibratory impulse as the collision of the skin against the leading land edge. Were frequency the most important variable affecting perceived roughness, land width and groove width should affect roughness in the same way. However, roughness increased with increasing groove width but decreased with increasing land width. Thus, vibratory frequency, per se, does not seem to be a significant contributor to perceived roughness.

There is a second way to test the energy vs frequency issue. Frequency of vibration can be manipulated by holding the surface geometry constant, and instructing the $S$ to move his hand at different touching speeds. Katz (1925) wrapped a long strand of copper wire (.25-mm diam) around a pencil to form a series of ridges. The $S$ moved his finger across the ridged surface using a 10 -fold range of speeds $(1-10 \mathrm{~cm} / \mathrm{sec})$. Subjectively, the roughness of the wrapped pencil remained essentially unchanged, suggesting that, within this limit, Ss were able to compensate for changes in vibratory frequency by taking velocity into account. With yet higher speeds, the surface felt smoother, and finally painful (p. 231).

The method of altering hand velocity was used in the present experiment. When hand-speed increases, there is more skin deformation per second, and so the total vibratory energy/second also increases. This assumes that (i) energy depends upon some aspect(s) of skin deformation, and (ii) the vibratory energy per deformation of skin in a single groove remains constant across the vibratory frequency spectrum. The relation between energy and deformation is not known at this time, and will not be discussed further. If vibratory frequency is an important parameter, perceived roughness should change as the rate of hand motion is altered. If, however, energy/skin deformation in a groove is most influential, there should be little change in the apparent roughness of the grooved surfaces.

\section{Method}

In the present experiment, two parameters were systematically varied, viz, groove width (see Table 1) and rate of hand motion. Finger force was maintained at $1 \mathrm{oz}(\approx 28 \mathrm{~g})$ throughout.

Two Hunter timers in series with a neon light served as a "metronome." Although the apparatus had not been designed for this purpose, the two loud clicks produced by the timer relays functioned satisfactorily as a signal which $\mathrm{Ss}$ could use to control their rate of hand motion. The period between the clicks could be adjusted using the timer controls.

The Ss were trained to move their fingers over the grooved section of the plate in time with the clicks. A procedure akin to "shaping" was used with each $S$ to attain the desired finger speeds and motions. $S$ was told that his finger would be placed on the smooth section of the plate, as in earlier experiments, but this time at the edge of the central section. His task was to move his finger across the plate between clicks, each pass just covering the central section of the plate. It was explained that the reason for doing this was to ensure that a given distance was travelled in the period of time specified by the clicks. In this way, a given rate of motion could be fairly well assured. By trial and error, the extreme rates which $S$ s could maintain reasonably well were determined. The rates finally used were approximately $0.4,2$, and $10 \mathrm{in./sec} \mathrm{(about} 1,5$, and $25 \mathrm{~cm} / \mathrm{sec}$ ). For purposes of analysis and discussion, they will be referred to henceforth as "slow," "medium," and "fast" rates, respectively. At least 4 days of practice were spent on the task before the experiment proper was begun. By this time, $S$ agreed he was comfortable judging the roughness of the plates while maintaining the proper speeds. Before this, it had been difficult for him to concentrate on both the surfaces and the correct timing. During both training and experimental sessions, $S$ listened to the clicks through earphones, since extraneous noise could have interfered with what was already a difficult task. The timing of the interval between clicks was adjusted for the next plate immediately after $S$ had responded, so that he could listen to the clicks while $\mathbf{E}$ was changing the plate. By the time the actual experiment was begun, $S$ had become quite familiar with the timings. Each $S$ repeated the stimulus conditions twice a day for 5 days, with 1 day of practice at the beginning.

\section{Results}

An analysis of variance showed that rate of hand motion significantly affected perceived roughness $(p<.025)$. The results are shown in Fig. 3, where perceived roughness (log manitude estimate) is plotted against groove width for the three rate conditions. Each datum is the geometric mean of 60 observations. The shape of the function is concave upwards in each case. As in earlier studies, perceived roughness generally increases with increasing groove width. The apparent roughness of the plates examined at slow and medium rates of hand motion were very similar. At high speed, the roughness decreased slightly. There is also a decreasing contrast between the rate conditions as groove width increases, although the Groove Width by Rate interaction term was not significant. Returning to the simple effect of rate, one may observe that, although this result was statistically significant, the change in perceived roughness was no more than $1 \mathrm{~dB}(1 \mathrm{~dB}$ is equivalent 
to $1 / 10$ th of $1 \log$ unit) increase in perceived roughness for a 25 -fold change in hand speed. Such an effect is small when compared to the general findings (see Figs. 1 and 3; also Experiment IV) of a 4-dB increase in perceived roughness per doubling of groove width (the relatively flat portion at the bottom of the roughness functions is ignored for reasons explained in Experiment IV), or a 1-2-dB change for a 9-fold alteration in finger force (Lederman, 1973). The small effect that is obtained might be accounted for by the slight reduction in vibratory energy/deformation, to be expected as rate of motion is increased. The skin passes in and out of a groove less (especially for narrow grooves), because it does not have as much time to descend into the groove. A more detailed discussion of vibratory energy as it relates to skin deformation and perceived roughness is presented in a paper by Taylor and Lederman (1974). It appears again that vibratory frequency plays very little role in the perception of surface roughness by "relatively active" touch.

One may also consider the results in terms of the role of rate of hand motion as a component of the touching process. The range of hand speeds in this experiment was approximately $1-25 \mathrm{~cm} / \mathrm{sec}$, compared to the range of $1-10 \mathrm{~cm} / \mathrm{sec}$ used by Katz (1925). Where the speed ranges of the two studies overlap, i.e., $4 \mathrm{in} . / \mathrm{sec}(1 \mathrm{~cm} / \mathrm{sec})$ and $2 \mathrm{in.} / \mathrm{sec}$ $(5 \mathrm{~cm} / \mathrm{sec})$, the perceived roughness is relatively unaffected by rate of motion. The largest shift in the present experiment, although slight in relative terms, occurred when the high rate was used, and was in the direction mentioned by Katz. There is thus a strong constancy for roughness with variation in hand speed when the latter is varied by the $O$. This contrasts with the results of an experiment performed by Katz (1925) in which paper surfaces varying in roughness were moved across an O's passive hand at speeds of 3,15, and $60 \mathrm{~cm} / \mathrm{sec}$. At the highest speed, all papers seemed much smoother and less discriminable than at the medium speed; at the lowest speed, "...the smoother papers seemed to become less smooth..." than with the middle speed, and again were less discriminable.

Worth mentioning at this point is an experiment (Lederman, 1973) in which Ss judged the roughness of grooved plates of different groove widths (with land width constant) at two different hand forces [ 1 and $9 \mathrm{oz}(\approx 18$ and $\approx 252 \mathrm{~g})]$ and the medium and fast speeds used in Experiment II. The same very small rate effects were obtained, but no significant interaction between speed and force was found. It would appear that under these experimental conditions, the two components of the touching process studied in Experiments I and II function independently of one another in the perception of roughness.

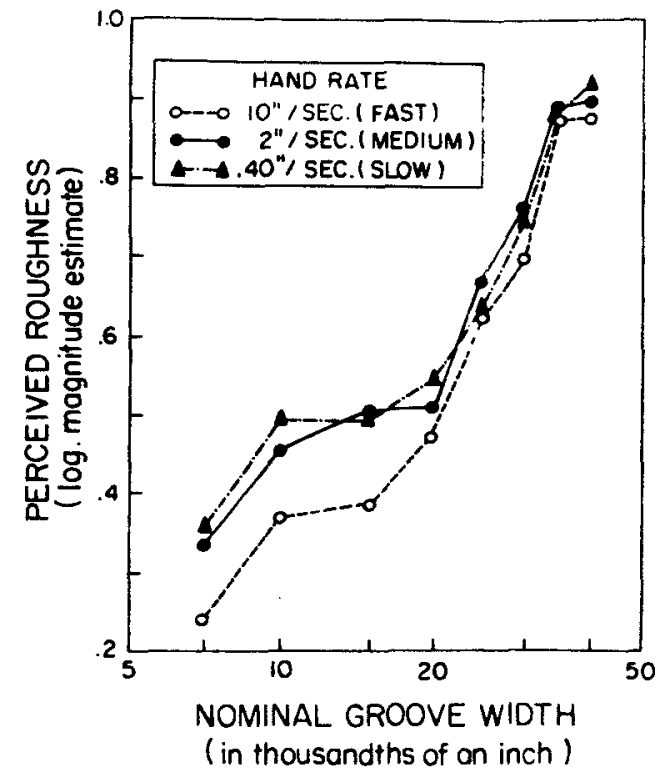

Fig. 3. Experiment II: Perceived roughness as a function of groove width and rate of hand motion. Fingertip force is $1 \mathrm{oz}$ $(\approx 28 \mathrm{~g})$.

\section{MACROSCOPIC SURFACE STRUCTURE}

\section{Experiment III}

The Lederman and Taylor (1972) study is the first in a series of experiments investigating the effects of surface geometry, i.e., the planned "macroscopic" alterations in surface structure. Together with the results of a study by Lederman (1973), the following data have been obtained. Perceived roughness of grooved surfaces tends to increase with increasing groove width, and to decrease in a reasonably linear fashion with increasing land width. However, on its own, spatial wavelength, i.e., 1 land +1 groove, has no effect on perceived roughness. This result has been verified in two different ways. The first has been described in Experiment II, where the Lederman and Taylor (1972) experiment was discussed in relation to the vibratory frequency-energy issue. A constant spatial wavelength was maintained between corresponding pairs of plates in the two sets described. If spatial wavelength was important, growth curves of roughness as a function of groove and land width should have been quite similar. However, the two parameters affected the perception of roughness in opposite directions. A second method (Lederman, 1973) of assessing the significance of spatial wavelength involved the use of a set of plates in which spatial wavelength was held constant, while the groove and land widths were adjusted accordingly. Once again, the roughness estimates changed, indicating failure to find an important spatial wavelength effect. Using the same technique, Lederman (1973) also 

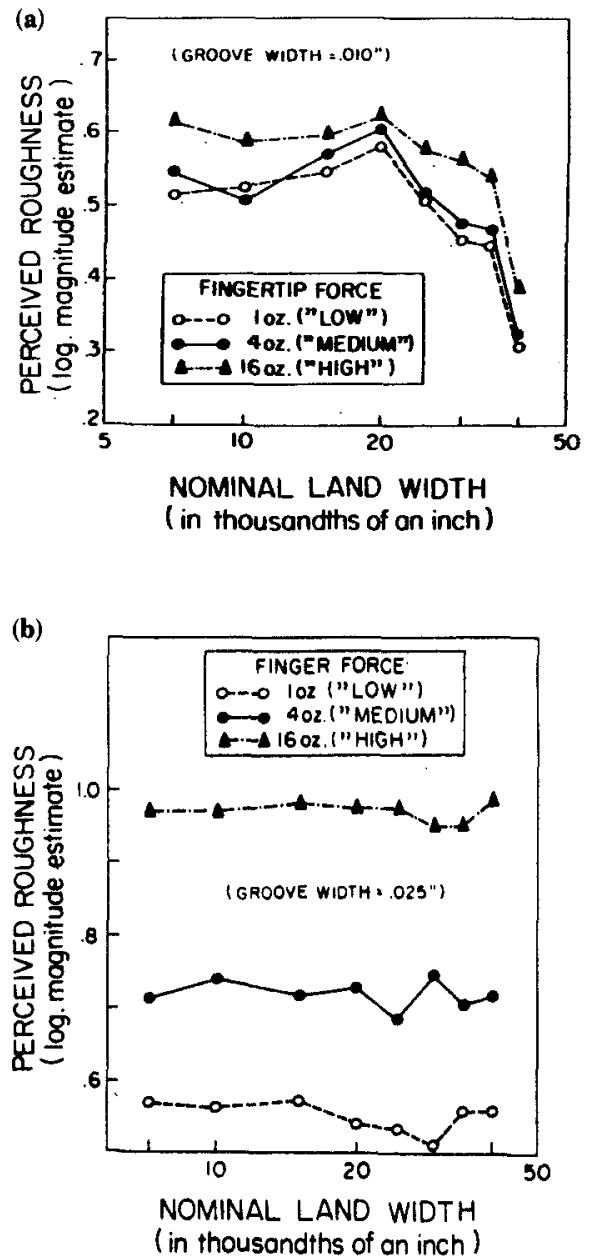

Fig. 4. Experiment III: (a) Percelved roughness as a function of land width and controlled fingertip force [groove width $=.010$ in. $(\approx .25 \mathrm{~mm})]$. (b) Perceived roughness as a function of land width and controlled fingertip force [groove width $=.025 \mathrm{in}$. $(\approx .625 \mathrm{~mm})]$.

found that groove-to-land ratio is not a significant variable in the perception of roughness.

Experiment III continues the investigation of surface geometry and its effects on apparent roughness. So far, groove width has proved far more important than land width. Would even this slight decrease in perceived roughness with increasing land width occur when a large constant groove width was used? Or would the effect of the wider groove override any smaller effect due to land width?

Two sets of plates were used to examine these questions. The sets varied similarly in land width, but the members of one set had a relatively narrow constant groove width and the members of the other, a relatively wide one. Ss were run for 7 days plus one practice session. Three fingertip force conditions were employed: 1,4 , and $16 \mathrm{oz}(\approx 28, \approx 112$, and $\approx 448 \mathrm{~g})$.

An analysis of variance indicated that the difference between the groove widths was not significant, in contrast to the highly significant values usually obtained (e.g., Lederman \& Taylor, 1972;
Experiments I, II; see also Experiment IV). This anomaly was due to the Subjects by Groove Widths term, which was highly significant $(p<.001)$ : the significance seemed due to one $S$, who showed a larger average difference between the wide- and narrowgroove sets than did the other Ss. For each individual, however, the average perceived roughness of the wide-groove set was greater than that of the narrow-groove set.

The land width, force, and Land Width by Force terms were all significant $(\mathrm{p}<.05, \mathrm{p}<.005$, and $\mathrm{p}<.01$, respectively).

Figure $4 \mathrm{a}$ shows perceived roughness for the narrow-groove set as a function of land width for the three forces applied. Figure $4 \mathrm{~b}$ shows the corresponding results for the wide-groove set. The data may be directly compared because the Ss were told to keep the same range of numbers within a day's session. The differences in perceived roughness due to the force conditions appear to be much larger in the wide- as opposed to the narrow-groove set. Furthermore, the significant Groove Width by Land Width interaction is evident, because no appreciable land effect appears in the wide-groove set, while there is one, albeit small, for the narrow-groove set. This land effect is restricted to Plates L25 through L40. Over this latter section of the stimulus range, apparent roughness seems to decline as land width increases. The results of the present experiment do not replicate the linear effect of land width found in earlier studies (e.g., Lederman \& Taylor, 1972; Lederman, 1973). A possible explanation of the "land effect," as well as an interpretation of the difference across experiments will be reserved until the end of Experiment IV, since each requires consideration of microscopic irregularities on the plate surfaces.

\section{MICROSCOPIC SURFACE STRUCTURE}

\section{Experiment IV}

Two different methods of grooving the plates have been used for the present experiments. One technique involved the use of a machine known as a shaper. Parallel linear grooves are produced in the surface of a smoothed aluminum plate by means of a metal tool bit cut to groove-width specifications. The groove bottoms of plates produced in this way are slightly U-shaped. The shaper can cut a groove or land to within $\pm .002 \mathrm{in}$. $(\approx .05 \mathrm{~mm})$ of its nominal value. Plates cut in this manner will henceforth be referred to as S-plates.

The second technique is known as electric discharge machining (EDM), and may be used to produce plates which are approximately twice as accurate as the first method, i.e., lands and grooves are cut to within \pm .001 in. $(\approx .025 \mathrm{~mm})$ of their nominal value. The technique involves submerging in an oil bath a plate and electrode (carbon or tungsten) cut to specified groove width. By passing a dc current through the electrode, a spark is produced which cuts into the 
aluminum surface. The width of the groove cut is determined by the shape of the electrode and the depth by the size of the gap between plate and electrode. In contrast to the S-plates, the groove depth of each of the EDM plates was made equal to or greater than the groove width. This was done to prevent any chance of the finger's "bottoming" on the groove floor.

It was quite apparent that the texture of the EDM-plates felt somewhat "different" from that of the S-plates. Moreover, scanning electron microscopy ${ }^{3}$ performed on sections of the two kinds of plates revealed microscopic differences in the physical structure of the lands. A scanning electron micrograph (Fig. 5) of a small sample cut from S-plate GB5 shows a regular "railway track" surface extending continuously along the edges of the S-plate surface. As burring of the edges had been originally expected with the shaping procedure, all S-plates were burnished after cutting, but, as Fig. 5 indicates, this final process did not smooth the lands completely. Figure 6 is a micrograph of an EDM-plate [groove width $=.050$ in. $(\approx 1.25 \mathrm{~mm})$; land width $=.010 \mathrm{in}$. $(\approx .25 \mathrm{~mm})]$. Here, a different form of surface irregularity may be seen on the lands. Bumps of aluminum which have melted and subsequently hardened are irregularly distributed along the land edges of the EDM-plates.

With both physical and undefined perceptual differences existing between nominally identical plates sets, it was important to ask whether the apparent tactile roughness also differed. Accordingly, Experiment IV was designed to determine whether results obtained earlier, with the set of S-plates which varied in groove width (Experiment I), would be replicated when Ss were presented with a set of EDM-plates under corresponding groove width and force conditions. From Table 1, it can be seen that G5, the plate with the narrowest groove width [.005 in. $(\approx .125 \mathrm{~mm})]$ was an S-plate. It was not possible to produce a groove width narrower than $.007 \mathrm{in.}(\approx .175 \mathrm{~mm})$ using the EDM technique. Hence, to equate the set of groove widths with that from Experiment I, the corresponding S-plate was employed.

The results are shown in Fig. 7. Perceived roughness is plotted as a function of groove width at each of the three finger forces. The results at first sight appear quite similar to those in Fig. 1. The three functions may be described in two portions, one relatively flat portion for narrow grooves, where roughness is independent of groove width, and one for wider grooves, where roughness is linearly related to $\log$ groove width. Using a least-squares fit (solid lines), the slopes of the rising portions of the functions were found to be $1.43 \pm .22,1.38 \pm .07$, and $1.72 \pm .12$ for the low, medium, and high forces, respectively. In determining these slopes, the data which describe the flat portion (Plates G5, G10, and G15 for the low force condition and Plates G5 and

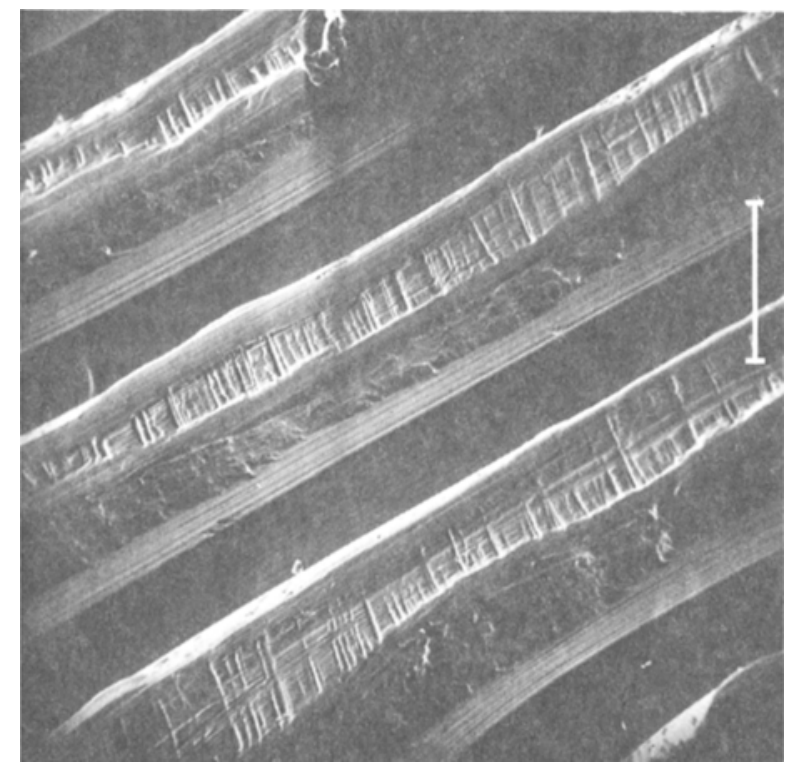

Fig. 5. Scanning electron micrograph of the land surface on an S-plate [groove width $=.005 \mathrm{ln} .(.125 \mathrm{~mm})$; land width $=.010 \mathrm{in}$. $(\approx .25 \mathrm{~mm})]$. The "railway tracks" may be seen on the top of the lands. The thin white line represents a length of 200 microns. The darker areas represent the grooves.

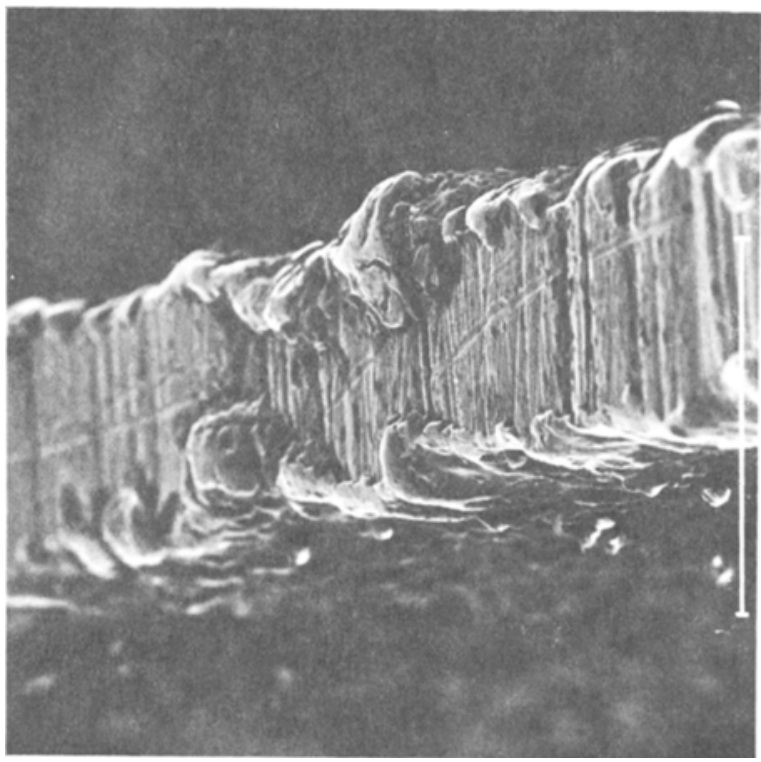

Fig. 6. Scanning electron micrograph of the land surface of an EDM-plate [groove width $=.050 \mathrm{ln} .(\approx 1.25 \mathrm{~mm})$; land width $=$ .010 in. $(\approx .25 \mathrm{~mm})]$. The raised mounds of hardened alnminum may be seen irregularly scattered along the edges of the land. The darker areas represent the grooves. The thin white line represents a length of 200 microns. 


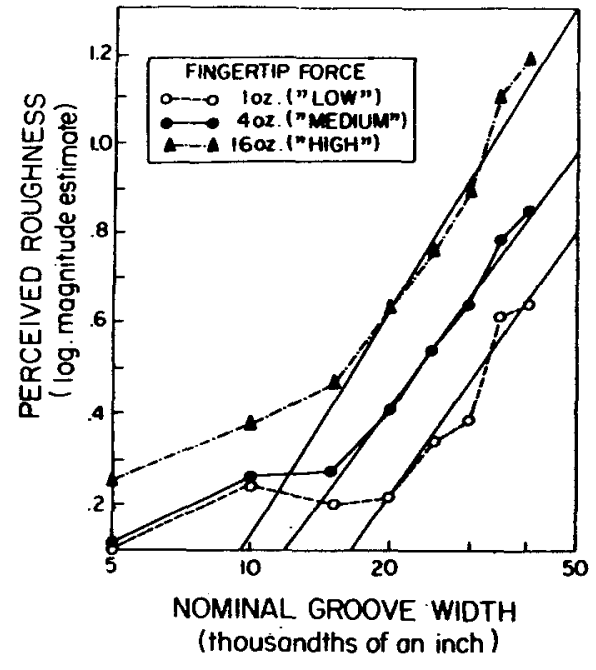

Fig. 7. Experiment IV: Perceived roughness as a function of groove width and controlled finger force. All plates with the exception of G5 are EDM-plates.

G10 in the other two force conditions) were omitted. The reason for this omission is discussed in the next section. The steeper slope for the high force condition is due to high roughness values for $\mathrm{G} 35$ and G40, and may be anomalous.

It is suggested that the microscopic surface irregularities (shown in Figs. 5 and 6) can account for the constant roughness of the narrow-groove plates, the land effect, and several discrepancies between the EDM- and S-plate data which appear on closer observation. The discussion of discrepancies will extend to the data from Experiment III, since the two land-varying sets were also EDM-plates, and then compare these results to the S-plate data from earlier experiments (Lederman \& Taylor, 1972; Lederman, 1973).

The irregularities on the land edges might account for the relatively lower slope of the roughness function at the narrow-groove ends of the low-force functions (Experiments I, II, and III). It is possible that irregularities in the nominally uncut sections of both kinds of plates masked the additional roughness due to the cuts. For the narrowest grooves, the roughness estimates might refer to the basic finish of the lands rather than to the increasingly finer groove sections. Presumably, such masking would decline with increasing force as the finger pushed deeper into the groove [the role that depth of skin deformation plays in the perception of roughness is discussed in greater detail in Taylor and Lederman (1974)]. Figures 5 and 6 lend support to the physical possibility of masking. An experiment should be designed to test the implications of a masking interpretation in terms of microscopic irregularities on the land edges. One would predict that masking would not occur with perfectly smooth lands, and therefore that perceived roughness would continue to decrease at the same rate with decreasing groove width until a true threshold was reached.

The land effect, i.e., the inverse relation between land width and perceived roughness (Lederman \& Taylor, 1972; Experiment II) may also be accounted for by the irregularities of the plate surface structure. Figures 5 and 6 indicate ridges on the land surfaces, at or near the groove edges of both the EDM- and S-plates. The narrow lands would have proportionately more of an irregular surface than the wider lands. If true, the roughness of such plates would be enhanced relative to those with wider uncut portions between the grooves.

How do the EDM- and S-plate data compare, and can we account for the differences which appear in the data by differences in the two production techniques? The EDM constant roughness portion (e.g., Experiment IV, Fig. 7) occurs at a somewhat higher roughness value, and intersects the rising portion of the curve at a wider groove value than the corresponding section of the S-plate function (Experiment I, Fig. 1). Different skin deformation patterns must occur because of differences in the EDM- and S-plate microsurface structure. In the former, the skin is stretched in at least two directions, i.e., across the land surface between adjacent ridges for a pair of groove edges, and along the land between irregularly raised sections of a single groove edge (Fig. 6); for the latter, the skin deformation is unidirectional between the "railway tracks" (Fig. 5). If masking is positively related in some undefined fashion to this multidirectional aspect of skin stretch, the EDM-plate finish may be judged as rougher. The increased masking for the EDM-plates relative to the S-plates would therefore account for the difference in roughness values at the low end of the groove scale and the higher intersection point of the two portions of the growth curves described in the EDM- and S-plate data above.

A second discrepancy concerns the difference in the narrow-groove land effect as found in the Lederman and Taylor (1972) study (Fig. 5b) and in Experiment III (Fig. 4a). In the former experiment, the roughness function is described by a straight line, but in the latter, the curves are concave downwards. Conceivably, different amounts of masking due to. differences in the finish on the lands (Figs. 5 and 6) may account for the discrepancy. The increased masking would sum with the previously interpreted linear land effect to flatten or reduce the latter over the narrow land portion of the EDM-plate scale.

Finally, we must consider one last discrepancy between the roughness estimates for the EDM- and S-plates. Large differences occur among the force conditions when the wide-groove (land-varying) set is used in Experiment III (Fig. 4b), but not on presentation of the narrow one. The explanation offered above, i.e., masking by surface irregularities, may account for such differences in the following way. 
In an experiment by Lederman (1973), the roughness values assigned to $\mathrm{S}$-plates in a narrow-groove, land-varying set fell close to those for the narrow grooves of a groove-varying set. The dimensions of this land-varying set are those of the narrow-groove set in Experiment III, while the groove-varying set was GA of Experiment I (see Table 1). At narrow groove widths, where masking has been postulated, we find this correspondence of roughness estimates. Furthermore, it has been suggested that the masking effect is greater when EDM-plates are presented, as in Experiment III. If such masking occurred in this experiment, the finger probably bottomed on the masking irregularities. The greater contribution of the EDM irregularities to the final roughness estimates, then, should result in there being little, if any, effect due to finger force. There is no reason to assume that masking should dominate the perceived roughness when the groove widths are relatively wide. Therefore, the full force effect should occur in the wide-groove, land-varying set (Fig. $4 \mathrm{~b}$ ).

From the discussion above, it becomes clear that any complete account of the perceived roughness of grooved surfaces must consider not only macroscopic structure, but also the microscopic irregularities on the grooved surface. The need for this new level of analysis further serves to emphasize the importance of developing highly precise stimulus production techniques. Unfortunately, such techniques for producing tactile stimuli within a limited budget do not yet exist.

\section{TOWARDS A MECHANICAL ANALYSIS OF SKIN DEFORMATION}

The four experiments reported provide a data base for an analysis of tactile roughness of grooved plates in terms of static deformation of the skin touching the surface (Taylor \& Lederman, 1974). Dynamic aspects of skin deformation were not considered because of the relatively negligible effects of a 25 -fold change in rate of hand motion (Experiment II).

The major parameters of perceived roughness in the present experiments were the groove width and applied fingertip force (regardless of whether the $E$ or the $S$ chose the force value). Considering the linear sections of the force curves in Experiment IV (Fig. 7), doubling groove width appears to be equivalent in terms of changes in perceived roughness to multiplying finger force by $16\left(2^{4}\right)$, i.e., $F^{4} \equiv G$. This equivalence relation holds for the $S$-plate functions (Fig. 1) as well. Thus, roughness may be described as increasing approximately as $\mathrm{f}\left(\mathrm{FG}^{4}\right)$ for grooves over $.015 \mathrm{in} .(\approx .375 \mathrm{~mm})$, where $\mathrm{f}$ is the function $\mathrm{k}\left(\mathrm{FG}^{4}\right)^{1 / 4}$ and $k$ is an arbitrary constant. Three skin deformation parameters were found to predict roughness as a function of finger force and groove width quite well. They are the depth to which the finger descends in the groove, the cross-sectional area of the finger within the groove, and the cross-sectional area of the deviation of the skin from its resting position. The last parameter also qualitatively predicts the effect of land width on perceived roughness, and is therefore tentatively favored as the "stimulus" for perceived roughness.

\section{REFERENCES}

Ekman, G., Hosman, J., \& Lindström, B. Roughness, smooth ness and preference: A study of quantitative relations in individual subjects. Journal of Experimental Psychology, 1965, 70. 18-26.

Gibson, J. J. The senses considered as perceptual systems. Boston: Houghton Mifflin, 1966.

KATz, D. Der A ufbau der Tastwelt (The world of touch). Leipzig: Barth, 1925.

KRuEGER, L. David Katz's Der Aufbau der Tastwelt (The world of touch) A synopsis. Perception \& Psychophysics, 1970, 7. 337.341 .

LeDERMan, S. J. The perception of surface roughness by touch. Unpublished doctoral dissertation, University of Toronto, 1973.

Lederman, S. J., \& TAYlor, M. M. Fingertip force, surface geometry, and the perception of roughness by active touch. Perception \& Psychophysics, 1972, 12, 401-408.

Mennes, M., \& Zigler, M. J. An experimental study of the perceptions roughness and smoothness. American Journal of Psychology, 1923, 34, 542-549.

Stevens, S. S., \& Harris, J. R. The scaling of subjective roughness and smoothness. Journal of Experimental Psychology, 1962, 64, 489-494.

TAylor, M. M., \& Lederman, S. J. Tactile perception of grooved surfaces: A model and the effect of friction. Perception \& Psychophysics, 1974, in press.

Taylor, M. M., Lederman, S. J., \& Gibson, R. H. Tactual perception of texture. In E. Carterette and $M$. Friedman (Eds.), Handbook of perception. Vol. III. New York: Academic Press, 1973. Pp. 251-272.

Zigler, M. J. David Katz's Der Aufbau der Tastwelt. Psychological Bulletin, 1926, 23, 326-336.

\section{NOTES}

1. Since the machine shop works in the British system of measurement, the stimulus specifications and experimental designs were originally planned in these units and similarly reported here. Metric equivalents are included in parentheses.

2. If $\mathrm{Ss}$ are influenced by instructions, it should occur most strongly during the first session when they are new to the task. Subsequent investigation of the first sessions of numerous experiments in which ranges were similarly exemplified revealed that only a fraction of a percent of Ss used the suggested numbers or, for that matter, a range of 1 log unit. It seems safe to conclude therefore that the instructions do not bias Ss in this respect.

3. I would like to thank Ken Wright of the Department of Parasitology and Anne Kerr of the Department of Metallurgy at the University of Toronto for their assistance in producing the micrographs.

(Received for publication February 25, 1974; revision received June 26,1974 .) 